\title{
LAMP assays of Zika virus and other infectious agents will inevitably see expanded use due to their simplicity, sensitivity, specificity, and economy
}

\author{
Connie D. Brewster ${ }^{1}$, Nunya Chotiwan ${ }^{1,2}$, Rushika Perera ${ }^{1,2}$, Sandra L. Quackenbush ${ }^{1}$ Joel Rovnak $^{1}$ \\ ${ }^{1}$ Department of Microbiology, Immunology and Pathology, ${ }^{2}$ Arthropod-borne Infectious Disease Laboratories, College of Veterinary Medicine and \\ Biomedical Sciences, Colorado State University, Fort Collins, Colorado, USA \\ Correspondence to: Joel Rovnak, PhD. Department of Microbiology, Immunology and Pathology, College of Veterinary Medicine and Biomedical \\ Sciences, Colorado State University, Fort Collins, Colorado 80523, USA. Email: joel.rovnak@colostate.edu. \\ Provenance: This is an invited article commissioned by Section Editor Dr. Hui Kong (Department of Respiratory Medicine, The First Affiliated \\ Hospital of Nanjing Medical University, Nanjing, China). \\ Response to: Lustig Y, Sofer D, Hindiyeh M, et al. LAMP assay for specific detection of Asian and African lineage Zika virus: will it meet the \\ expectations? Ann Transl Med 2018;6:53.
}

Submitted Feb 11, 2018. Accepted for publication Feb 25, 2018.

doi: 10.21037/atm.2018.03.01

View this article at: http://dx.doi.org/10.21037/atm.2018.03.01

We would like to thank Annals of Translational Medicine for the opportunity to respond to the editorials on our recent publication, Rapid and specific detection of Asian- and Africanlineage Zika viruses. And we thank Yaniv Lustig, Danit Sofer, Musa Hindiyeh, and Ella Mendelson, and Erick Mora-Cárdenas and Alessandro Marcello for their carefully considered remarks. The editorials reflect the writer's experience in evaluating environmental samples for many diseases and show their great interest in the continuing development of diagnostic tools for deployment at points of care and in the field. We would like to take this opportunity to address their points, with which we are largely in agreement but may not have made clear in the publication.

We have currently deployed or support deployment of our Zika LAMP assay in Brazil, Cuba, Nicaragua, Hawaii, and Puerto Rico in order to identify technical problems that may have been overlooked in the laboratory, and we have successfully performed LAMP assays in the field without a cold chain using simple liquid handling equipment and a heated-lid hot block plugged into a vehicle battery. Our initial goal in the development of the assay was field deployment for vector surveillance, and we continue to refine the assay to make it as simple and accurate as possible in adherence to the World Health Organization's "ASSURED" guidelines. In the laboratory, we have tested and newly incorporated existing technologies, such as alternative dyes to improve readout and contamination control. The latter includes uridine-5'-triphosphate (UTP) and uracil-DNA glycosylase (UDG) in reaction mixes, which we highly recommend. Although our assessments of reagent stability were not included in the publication, we had done and continue to evaluate prepared reaction mixtures for failure rates after storage at ambient and elevated temperatures for increasing periods of time. We have found prepared reagents to be remarkably robust without a cold chain. We arrived at these determinations with no competing interests or support from commercial suppliers of LAMP reagents.

As described in our publication, the basic LAMP assay does not require prior reverse transcription, and, while the enzyme has been described as having "reverse transcriptase activity", it is likely to have mechanisms that are quite distinct from reverse transcriptase, particularly in its ability to use both RNA and DNA templates continually during the reaction. A goal of the paper was to establish this capacity of the Bst polymerase and to end the perception of a requirement for RT. This an important advantage over reverse transcriptase-polymerase chain reaction (RT-PCR). Another goal was to establish the utility of crude extracts for nucleic acid detection with LAMP. This is true for both LAMP and RT-PCR, as presented in the data. RNA isolation adds a significant burden of cost and time to both RT-PCR and 
LAMP protocols. However, it does increase the sensitivity of both assays. This is an important point in understanding the utility of LAMP: it is as sensitive and as specific as RTPCR when assaying purified RNA preparations, and it is more sensitive and more specific than RT-PCR when assaying crude extracts. LAMP is the choice for any conditions that preclude the use of laboratory facilities for RNA isolation and thermal cycling. However, such applications come at a cost, as defined in the publication, in terms of sensitivity and specificity, and this must be carefully considered when evaluating the results. On the other hand, where laboratory facilities for RNA isolation and thermal cycling are available, LAMP is comparable to RT-PCR, but at lower cost per sample due to the exclusion of the RT step. Thermal cycling instruments with melt curve analysis also make it possible to perform multiplex LAMP assays by distinguishing the melting points of various products in the same reaction. LAMP and RT-PCR suffer the same inherent constraint: they require nucleic acid replication that is associated with an active infectious process. That means detection during the viremic phase of acute infection for flaviviruses and many other viruses. In the case of Zika virus, nucleic acid detection during convalescence is possible in body fluids such as semen which may have persistent virus RNA, but serological assays remain the standard for convalescent diagnoses.

During the preparation of the manuscript, sequence-based analyses of Zika virus lineages were newly available, and this information guided both our LAMP primer selection and our conclusions regarding the future utility of assays that distinguish African from Asian strains. The sequences of these lineages are quite divergent and, while there were no known differences in pathology or infectivity at the time, we felt it prudent to distinguish them in anticipation of inevitable overlaps of active infections by viruses of both lineages in the same geographic region. Although infection by either strain is likely to confer immunity to the other, little is known of the current extent of African-lineage Zika virus infection or exposure rates in Africa. Naïve populations in Africa may be subject to renewed rounds of infection by Asian-lineage viruses with possible differences in disease outcomes. Since publication, it has become clear that the currently circulating Asian-lineage viruses do have accumulated substitutions that confer increased infectivity and neuropathology (1-3). After 2012, the Asian-lineage Zika viruses acquired a fixed mutation in the NS1 gene that enhances mosquito infection by inhibiting interferon- induction $(1,2)$, and a single amino acid substitution was identified in the prM protein that increases infectivity in human and mouse neural progenitor cells, increases microcephaly in mouse fetuses, and causes higher mortality in neonatal mice (3). We believe that a rapid and specific assay that can distinguish African from Asianlineage viruses will be an important component to addressing health care should the contemporary epidemic strains "reenter" Africa.

LAMP applications for a variety of infectious diseases are expanding rapidly, as are new innovations in commercially available reaction mixtures and enzymes, remote monitoring and mapping of results with handheld devices, and exothermic chemical heating systems for LAMP assays without electricity. We believe that LAMP assay development will continue to improve its utility, simplicity and frequency of application.

\section{Acknowledgements}

None.

\section{Footnote}

Conflicts of Interest: The authors have no conflicts of interest to declare.

\section{References}

1. Liu Y, Liu J, Du S, et al. Evolutionary enhancement of Zika virus infectivity in Aedes aegypti mosquitoes. Nature 2017;545:482-6.

2. Xia H, Luo H, Shan C, et al. An evolutionary NS1 mutation enhances Zika virus evasion of host interferon induction. Nat Commun 2018;9:414.

3. Yuan L, Huang XY, Liu ZY, et al. A single mutation in the prM protein of Zika virus contributes to fetal microcephaly. Science 2017;358:933-6.

Cite this article as: Brewster CD, Chotiwan N, Perera R, Quackenbush SL, Rovnak J. LAMP assays of Zika virus and other infectious agents will inevitably see expanded use due to their simplicity, sensitivity, specificity, and economy. Ann Transl Med 2018;6(10):196. doi: 10.21037/atm.2018.03.01 